\title{
Chromatin remodeling and histone modification in the conversion of oligodendrocyte precursors to neural stem cells
}

\author{
Toru Kondo ${ }^{1,2,3}$ and Martin Raff ${ }^{1}$ \\ ${ }^{1}$ Medical Research Council Laboratory for Molecular Cell Biology, Cell Biology Unit, and the Biology Department, \\ University College London, London WC1E 6BT, United Kingdom; ${ }^{2}$ Centre for Brain Repair, University of Cambridge, \\ Cambridge CB2 2PY, United Kingdom
}

\begin{abstract}
We showed previously that purified rat oligodendrocyte precursor cells (OPCs) can be induced by extracellular signals to convert to multipotent neural stem-like cells (NSLCs), which can then generate both neurons and glial cells. Because the conversion of precursor cells to stem-like cells is of both intellectual and practical interest, it is important to understand its molecular basis. We show here that the conversion of OPCs to NSLCs depends on the reactivation of the sox 2 gene, which in turn depends on the recruitment of the tumor suppressor protein Brca1 and the chromatin-remodeling protein Brahma (Brm) to an enhancer in the sox2 promoter. Moreover, we show that the conversion is associated with the modification of Lys 4 and Lys 9 of histone $\mathrm{H} 3$ at the same enhancer. Our findings suggest that the conversion of OPCs to NSLCs depends on progressive chromatin remodeling, mediated in part by Brca1 and Brm.
\end{abstract}

[Keywords: Oligodendrocyte precursor cells; neural stem cells; Sox2; Brca1; Brm; histone modification]

Received May 17, 2004; revised version accepted September 21, 2004.

Oligodendrocyte precursor cells (OPCs) are arguably the best-characterized precursor cells in the vertebrate central nervous system (CNS) (Barres and Raff 1994). They can differentiate in vitro into either oligodendrocytes or type-2 astrocytes (2As), depending on extracellular signals: thyroid hormone (TH), for example, promotes oligodendrocyte differentiation (Barres et al. 1994), whereas bone morphogenetic proteins (BMPs) promote 2A differentiation (Mabie et al. 1997). Furthermore, if purified OPCs are first induced by BMPs to develop into 2As and are then cultured in basic fibroblast growth factor (bFGF), they convert to multipotent neural-stem-like cells (NSLCs), which can self-renew and produce neurons and type- 1 astrocytes, as well as oligodendrocytes and 2As (Kondo and Raff 2000a). The conversion to $2 \mathrm{As}$ seems to be a necessary first step in reprogramming OPCs to become NSLCs: if OPCs are freshly purified from the developing rat optic nerve and cultured directly in bFGF without an initial exposure to BMPs, they do not convert to NSLCs (Kondo and Raff 2000a). Since OPCs are multipotent (Kondo and Raff 2000a), present

${ }^{3}$ Corresponding author.

E-MAIL tk294@cam.ac.uk; FAX 44-1223-334121.

Article and publication are at http://www.genesdev.org/cgi/doi/10.1101/ gad.309404. throughout the adult CNS, and are stimulated to proliferate by CNS damage (Dawson et al. 2003), they are an attractive endogenous source of cells for CNS repair, especially if one could learn how to control their behavior in vivo.

In the present study, we examine changes in the expression of several candidate genes during the conversion of OPCs to 2 As and NSLCs. We show that the treatment of OPCs with BMP2 rapidly induces the expression of several genes known to be expressed in at least some neural stem cells (NSCs), including sox2, which encodes a member of the SRY-related, HMG-box-containing, transcription factor family (Gubbay et al. 1990). Sox2 is one of the earliest known transcription factors expressed in the developing neural tube, and there is increasing evidence that it is essential for the maintenance of NSCs (Zappone et al. 2000; Bylund et al. 2003; Graham et al. 2003). We demonstrate that depletion of Sox 2 by RNA interference (RNAi) blocks the proliferation of NSLCs and causes them to differentiate into neurons. We provide evidence that sox2 expression in NSCs and NSLCs depends on both Brcal and Brm, which is the catalytic subunit in a subset of SWI/SNF chromatin-remodeling complexes. Finally, we show that the conversion of OPCs to NSLCs is associated with the recruitment of Brcal and Brm to an enhancer in the sox2 promoter and 
that histone $\mathrm{H} 3$ in the enhancer is modified during the conversion to an active form, which is methylated at Lys 4 (K4) and acetylated at Lys 9 (K9). These findings suggest that the conversion is associated with extensive chromatin remodeling, which is mediated in part by Brcal and Brm.

\section{Results}

2As express several NSC markers

We first addressed whether the BMP-induced conversion of OPCs to $2 \mathrm{As}$ is associated with the expression of antigens that are expressed by at least some NSCs. We purified OPCs from postnatal day 6 (P6) rat optic nerve and expanded them for $4 \mathrm{wk}$ in PDGF, in the absence of $\mathrm{TH}$ to avoid their differentiation into oligodendrocytes (Barres et al. 1994). We then recultured the cells for $2 \mathrm{~d}$ on PDL-coated slide flasks, either in PDGF alone or in PDGF plus BMP2, and then fixed and immunolabeled them with the A2B5 monoclonal antibody (Eisenbarth et al. 1979), which labels rat OPCs (Raff et al. 1983); antiGFAP antibodies, which label astrocytes (Bignami et al. 1972) and some NSCs (Doetsch et al. 2002); and antibodies against Nestin, PSA-NCAM, LeX/SSEA1, or Sox2, all of which are expressed by at least some NSCs (Lendahl et al. 1990; Seki and Arai 1991; Li et al. 1998; Zappone et al. 2000; Capela and Temple 2002). Whereas $>90 \%$ of both OPCs and 2As expressed A2B5 and Nestin, no OPCs expressed GFAP, PSA-NCAM, SSEA1, or Sox $2 ;>90 \%$ of the BMP-induced 2As, however, expressed GFAP, $80 \%$ expressed SSEA1, 6\% expressed PSA-NCAM, and 60\% expressed Sox2 (Fig. 1A; data not shown). Thus, BMP2 rapidly induces many OPCs to express antigens that are characteristic of at least some NSCs.

\section{NSLCs and NSCs express similar mRNAs}

We next used RT-PCR to analyze several potentially relevant mRNAs in OPCs, 2As, NSLCs, and NSCs, including sox2, nestin, gfap, brca1, olig2, musashi1, hes1, hes5, bmi1, id1-4, and bcrp1 mRNAs, all of which have been shown to be expressed by at least some NSCs or by more restricted neural precursors. Olig2, hes1, and hes5 encode basic helix-loop-helix (bHLH) transcription factors (Akazawa et al. 1992; Sasai et al. 1992; Lu et al. 2000; Takebayashi et al. 2000; Zhou et al. 2000); musashi1 encodes an RNA-binding protein (Nakamura et al. 1994); bmi1 encodes a polycomb family transcription factor (van Lohuizen et al. 1991); id1-4 encode HLH proteins that inhibit cell differentiation (Benezra et al. 1990; Christy et al. 1991; Sun et al. 1991; Biggs et al. 1992; Riechmann et al. 1994); and bcrp1 encodes an ABC transporter expressed by various types of stem cells (Doyle et al. 1999).

OPCs were cultured in PDGF alone. We prepared 2As by culturing OPCs in PDGF plus BMP2 for $2 \mathrm{~d}$, and we prepared NSLCs by culturing the 2As in bFGF alone for 1 wk. We prepared NSCs from embryonic day 14.5

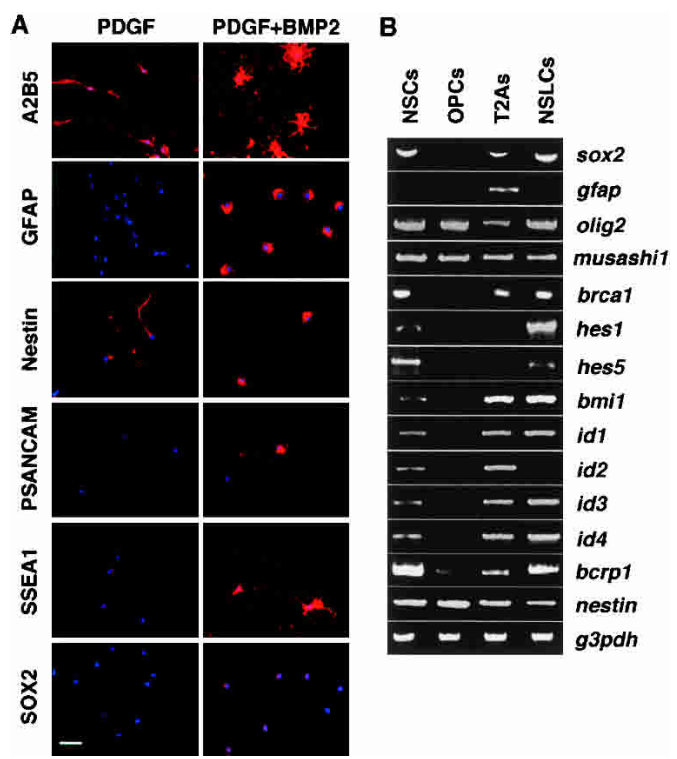

Figure 1. Expression of NSC-characteristic molecules in OPCs, 2As, NSCs, and NSLCs. (A) Purified P6 OPCs were expanded in PDGF without TH for $4 \mathrm{wk}$. They were then cultured in either PDGF (OPCs) or in PDGF plus BMP2 (2As) for $2 \mathrm{~d}$ and stained (in red) with the antibodies indicated. The cells were counterstained with Hoechst 33342 to visualize all nuclei (blue). Bar, $25 \mu \mathrm{m}$. (B) Purified OPCs were cultured as above for $4 \mathrm{wk}$ and then in PDGF alone (OPCs), in PDGF plus BMP2 for $2 \mathrm{~d}(2 \mathrm{As})$, or in PDGF plus BMP2 for $2 \mathrm{~d}$ and then in bFGF for 7 d (NSLCs). NSCs were prepared from E14.5 mouse brain by culturing the cells as floating neurospheres on uncoated dishes in bFGF for 2 wk. RNA was extracted and analyzed by RT-PCR, as described in Materials and Methods.

(E14.5) mouse telencephalon and expanded them in bFGF for 2 wk (Nakashima et al. 1999).

As shown in Figure 1B, NSCs expressed all of the examined mRNAs except gfap, whereas OPCs expressed only olig2, musashi1, and nestin mRNAs. (We showed previously that freshly isolated P0 rat OPCs express all four known mammalian Id mRNAs but that id4 rapidly declines as OPCs proliferate in vitro and in vivo [Kondo and Raff 2000b]; the present results indicate that the other three id mRNAs also eventually decrease as OPCs proliferate in culture and are no longer detectable after 4 wk.) The 2As expressed all but two of the examined mRNAs (hes1 and hes5, both of which encode effectors of Notch signaling), although olig2 mRNA decreased significantly compared to its expression in OPCs. When the 2As were cultured in bFGF for a week (to become NSLCs), they re-expressed both hes 1 and hes 5 mRNAs but lost gfap mRNA, and olig2 mRNA returned to the level seen in OPCs. Thus, a 2-d exposure to BMP2 induced a global change in gene expression in OPCs, and the patterns of expression in the mRNAs examined were similar in NSCs and NSLCs.

\section{NSLC proliferation and maintenance depends on Sox2}

As Sox2 is up-regulated when OPCs are treated with BMP2 (see Fig. 1), we investigated whether the depletion 
of Sox 2 by RNAi would influence the proliferation and/ or differentiation of NSLCs. We constructed expression vectors encoding either green fluorescence protein (GFP) alone (control) or GFP and a small interfering RNA specific for sox2 (sox2-siRNA). We first examined whether sox2-siRNA depleted endogenous Sox2. We transfected NSLCs with either the control vector or sox2-siRNA, cultured them in bFGF for $3 \mathrm{~d}$, and then fixed and immunolabeled them for both GFP and Sox2. Compared to the cells transfected with the control vector, the cells transfected with sox2-siRNA showed a greatly reduced expression of endogenous Sox2 (Fig. 2A).

We then transfected OPCs with either the control vector or sox2-siRNA, cultured them either in PDGF alone for $7 \mathrm{~d}$ (OPCs) or in PDGF plus BMP2 for $2 \mathrm{~d}$ and then bFGF for $5 \mathrm{~d}$ (NSLCs). We found that the NSLCs transfected with sox2-siRNA proliferated less than the NSLCs transfected with the control vector (data not shown), and we quantified this by adding bromodeoxyuridine $(\mathrm{BrdU})$ for $8 \mathrm{~h}$ and then staining the cells with anti-BrdU antibody; as shown in Figure $2 \mathrm{~B},>30 \%$ of the OPCs transfected with sox2-siRNA and $50 \%$ of the NSLCs transfected with the control vector incorporated BrdU, whereas none of the NSLCs transfected with sox2siRNA incorporated BrdU. Thus, the proliferation of NSLCs apparently depends on Sox2.

To determine whether the knock-down of Sox 2 by RNAi causes the spontaneous differentiation of NSLCs, we transfected OPCs, cultured them to produce NSLCs as above, and then fixed and immunolabeled the NSLCs for both GFP and a neural-specific antigen-either Nestin, GFAP, neurofilaments (NFs), which are specifically expressed by neurons (Julien 1999), or galactocerebroside
(GC), which is specifically expressed by oligodendrocytes (Raff et al. 1978). Whereas over $70 \%$ of the cells transfected with the control vector expressed Nestin, only $6 \%$ of the cells transfected with sox2-siRNA did so (Fig. 2C), suggesting that Sox2 is required to maintain Nestin expression in NSLCs. Similarly, whereas $10 \%$ of the cells transfected with the control vector expressed GFAP, none of the cells transfected with sox2-siRNA did so (Fig. 2D), suggesting that Sox 2 helps prolong the expression of GFAP as 2As convert to NSLCs in the presence of bFGF. In contrast, $40 \%$ of the cells transfected with sox2-siRNA expressed NFs, whereas none of the cells transfected with the control vector did so (Fig. 2E), suggesting that Sox2 normally inhibits the spontaneous differentiation of NSLCs into neurons. We could not detect any cells expressing GC among the cells transfected with either vector (data not shown), suggesting that Sox2 is not required to prevent the spontaneous differentiation of NSLCs into oligodendrocytes.

\section{Sox2 expression in NSLCs and NSCs depends on Brca1}

As Sox2 seems to play an important part in maintaining the proliferation and undifferentiated state of NSLCs, we investigated how sox2 expression is controlled in NSLCs and NSCs. There were two reasons for thinking that Brcal might be involved in this control, even though it was originally discovered as a tumor suppressor gene that predisposes women to breast and ovarian cancer (Miki et al. 1994). First, brca1 is expressed in the ventricular zone of the developing rodent brain, as well as in cultured embryonic and adult NSCs (Marquis et al. 1995; Korhonen et al. 2003). Second, brca1 ${ }^{-/-}$mice die as em-
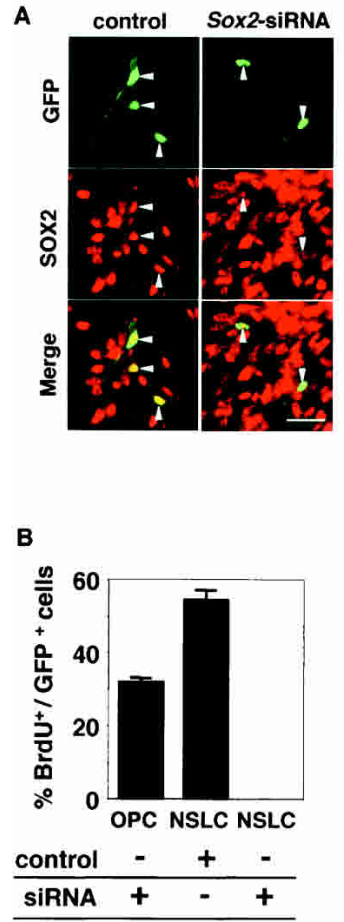
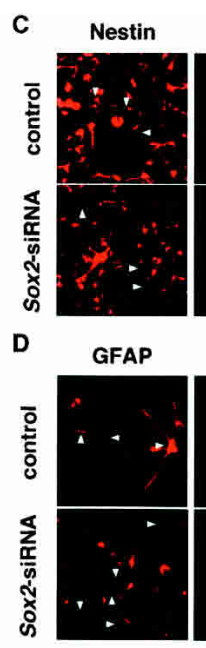

$\mathrm{E}$

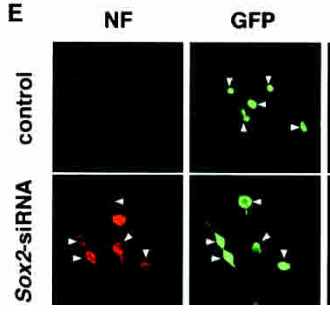

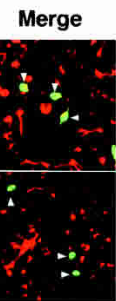
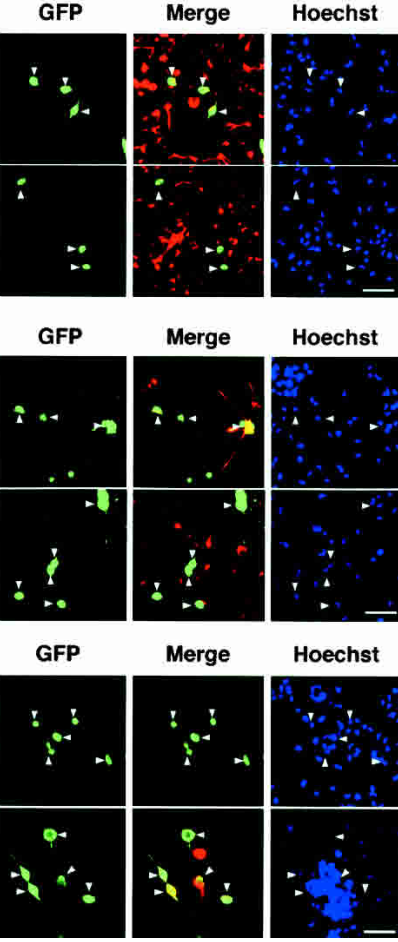

Hoechst

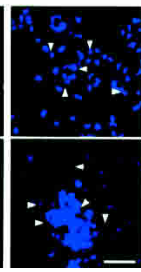

Figure 2. Effect of sox2-siRNA. (A) NSLCs were transfected with either a control vector encoding GFP alone or sox2-siRNA, cultured in bFGF for $3 \mathrm{~d}$, and then stained for both GFP (green) and Sox 2 (red). (B) OPCs were transfected as above and cultured either in PDGF alone (OPCs) or in PDGF plus BMP2 for $2 \mathrm{~d}$ and then bFGF for $5 \mathrm{~d}$ (NSLCs); BrdU was then added for $8 \mathrm{~h}$, and the cells were then fixed and immunolabeled for BrdU. The proportion of GFP-expressing cells that were $\mathrm{BrdU}^{+}$is shown as the mean $\pm \mathrm{SD}$ of three cultures. $(C-E)$ OPCs were transfected and cultured in PDGF plus BMP2 and then bFGF for $5 \mathrm{~d}$. The cells were then stained for both GFP (green) and the neural markers Nestin $(C)$, GFAP $(D)$, or NFs $(E)$-all in red. The cell nuclei were stained with Hoechst 33342 (blue). The GFP-positive cells are indicated with arrows. Bars: $A, C-E, 25 \mu \mathrm{m}$. 
bryos, with CNS defects that are very similar to those seen in sox2 $2^{-/-}$mice (Hakem et al. 1996; Liu et al. 1996; Avilion et al. 2003). We therefore examined the expression of Brcal protein in NSCs, OPCs, 2As, and NSLCs. As shown in Figure 3A, Brcal was not detectably expressed in OPCs but was expressed by 2As, NSLCs, and NSCs, indicating that its expression is upregulated when OPCs are induced to become $2 \mathrm{As}$ and NSLCs.

To determine the relationship between Brca1 and Sox2 expression, we constructed an expression vector encoding both GFP and a siRNA specific for brca1 (brca1siRNA). To show that brca1-siRNA could deplete endogenous Brcal, we transfected NSLCs with either the control vector or brca1-siRNA, cultured them in bFGF for $3 \mathrm{~d}$, and then fixed the cells and immunolabeled them for both GFP and Brcal. As shown in Figure 3B, transfection with brca1-siRNA significantly reduced the expression of endogenous Brcal. We then transfected NSLCs with either brca1-siRNA or sox2-siRNA, cultured them as just described, and immunolabeled them for either GFP and Brca1 or GFP and Sox2. Whereas all of the NSLCs transfected with sox2-siRNA still ex-

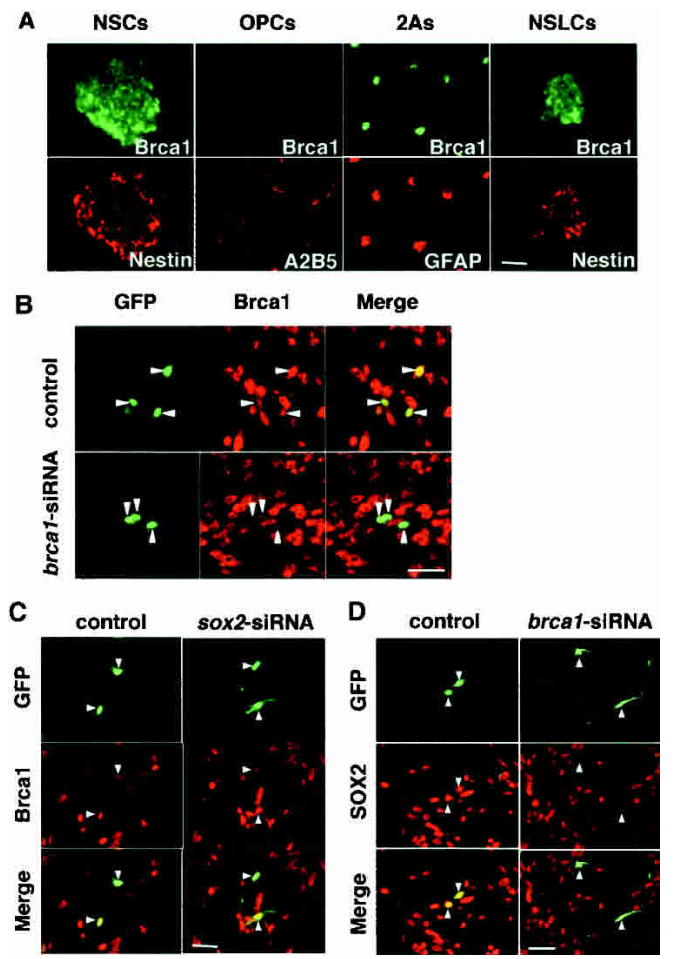

Figure 3. Role of Brcal in sox2 expression in NSLCs. (A) NSCs, OPCs, 2As, and NSLCs were immunostained for Brcal (green) and for Nestin, A2B5, or GFAP (in red). (B) NSLCs were transfected either with a control vector encoding GFP alone or with brca1-siRNA, cultured in bFGF for $3 \mathrm{~d}$, and then stained for GFP (green) and Brcal (red). (C,D) NSLCs were transfected with the control vector, sox2-siRNA, or brca1-siRNA, cultured in bFGF for $3 \mathrm{~d}$, and then stained for both GFP (green) and either Brcal or Sox2 (in red). Arrows indicate the GFP-positive cells. Bar: $A-D$, $25 \mu \mathrm{m}$. pressed Brcal (Fig. 3C), $>60 \%$ of the NSLCs transfected with brca1-siRNA did not express significant amounts of Sox2 (Fig. 3D). Transfection of cultured NSCs with brca1-siRNA resulted in a similar loss of Sox 2 expression (data not shown). These findings suggest that Sox2 expression in both NSCs and NSLCs depends on Brca1.

To determine if Brcal is sufficient to induce sox2 expression in OPCs, we transfected OPCs with an expression vector encoding both mouse Brcal and GFP, cultured them in bFGF for $7 \mathrm{~d}$, and fixed and immunostained them for GFP and Sox2. None of the GFP-positive cells were labeled for Sox2 (data not shown), suggesting that overexpression of brca1 on its own cannot induce Sox2 expression in OPCs; apparently, other factors are needed.

\section{Both Brca1 and Brm associate with an sox 2 enhancer} in cultured NSCs

To study the regulation of sox2 expression in our NSCs, we constructed a series of sox2-enhancer-luciferase expression vectors. We inserted the $5^{\prime}$-promoter region of the mouse sox2 gene, or various deleted forms (R1-R3) of the promoter, into the pGL3-promoter vector, which contains a minimal SV40 promoter upstream of a firefly luciferase cDNA (Fig. 4A). We transfected NSCs and 293 cells with these vectors, along with a vector (pEF-Rluc) encoding a sea pansy luciferase. After $24 \mathrm{~h}$, we assayed the firefly luciferase activity, normalized against the sea pansy luciferase activity. As shown in Figure 4A, both the full sox2 promoter and the R1-containing promoter in which R2 and R3 were deleted produced strong luciferase activity in NSCs, but both the R2-containing and $\mathrm{R} 3$-containing promoters in which $\mathrm{R} 1$ was deleted did not. These findings suggest that $\mathrm{R} 1$ contains an enhancer(s) for sox2 expression in cultured NSCs. In contrast, even the full promoter did not produce luciferase activity in 293 cells (Fig. 4A).

We then used chromatin immunoprecipitation (ChIP) to determine whether Brcal is associated with the R1 DNA sequence in NSCs. We fixed NSCs in formaldehyde, sonicated them in a cell lysis buffer, and harvested the extracts. We precipitated Brcal and its associated DNA from the extracts with anti-Brcal antibodies and Protein-A-Sepharose, purified the precipitated DNA fragments, and analyzed them by PCR. As shown in Figure $4 \mathrm{~B}$, we could detect the R1 but not the R2 or R3 DNA sequences, indicating that at least some Brcal is associated with the R1 sequence in the sox 2 promoter in NSCs and may thus be involved in stimulating sox2 expression in these cells.

Although Brcal is unlikely to bind directly to undamaged DNA, it has been shown to associate with SWI/SNF chromatin-remodeling complexes, which contain several subunits, including Brgl and Brm (Bochar et al. 2000). To determine if Brcal is associated with SWI/SNF complexes in NSCs, we did a ChIP analysis as just described, but using antibodies against Brm or Brg1; we also used antibodies against Brcal as a positive control 
A

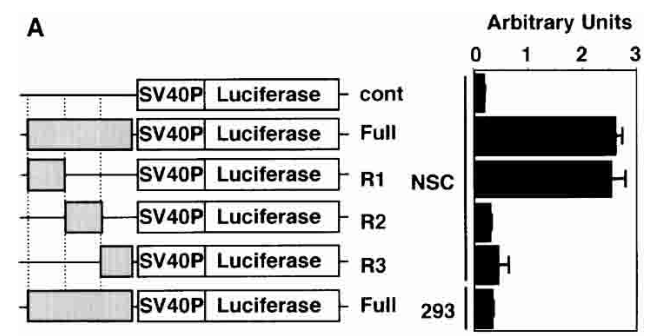

B

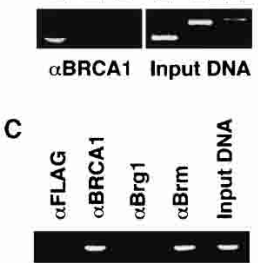

D

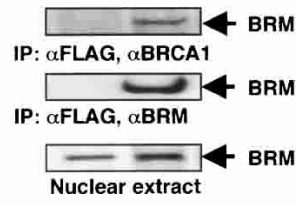

Figure 4. Association of Brcal and Brm on the R1 sox2 enhancer in NSCs. (A) NSCs or 293 cells were cotransfected with both pEF-Rluc, which encodes a sea pansy luciferase, and one of the sox2-enhancer-firefly-luciferase expression vectors containing the full-length or deleted versions of the sox2 promoter (shown on left). The activity of firefly luciferase was analyzed after $24 \mathrm{~h}$, normalized to the sea pansy luciferase activity. $(B)$ ChIP analysis to determine if Brcal is bound to the R1 DNA sequence in the mouse sox 2 promoter. Extracts of formaldehyde-fixed NSCs were immunoprecipitated with Brcal antibodies and Protein-A-Sepharose, and genomic DNA fragments in the immunoprecipitate were analyzed by PCR with specific primer sets to amplify the R1, R2, or R3 sequences. The gel positions of the R1, R2, and R3 sequences are shown on the right (input DNA). (C) ChIP analysis to determine whether the R1 coprecipitates with Brm or Brgl subunits of the SWI/SNF complex; anti-Brcal antibodies and anti-Flag antibodies were used as positive and negative controls, respectively. The gel position of the R1 sequence is shown at the right (input DNA). (D) Coprecipitation of Brcal and Brm proteins from extracts of NSCs. The extracts were immunoprecipitated with antibodies against Brca1, Brm, or Flag, and Protein-A-Sepharose, and the immunoprecipitates were analyzed by Western blotting using anti-Brm antibodies.

and against the Flag epitope as a negative control. As shown in Figure 4C, both the anti-Brcal and anti-Brm antibodies precipitated the $\mathrm{R} 1$ sequence, whereas the anti-Brg1 and anti-Flag antibodies did not, suggesting that Brcal is associated with Brm-containing SWI/SNF complexes in NSCs.

To confirm that Brcal and Brm are in the same protein complexes, we prepared extracts of NSCs and treated them with antibodies against Brcal, Brm, or Flag, followed by Protein-A-Sepharose, and then analyzed the immunoprecipitates by Western blotting using anti-Brm antibodies. As shown in Figure 4D, both the anti-Brcal and anti-Brm antibodies precipitated Brm, whereas the anti-Flag antibody did not, suggesting that at least some Brcal and Brm are in the same complexes in NSCs. Taken together, these results suggest that both Brcal and Brm associate with the R1 sox2 enhancer and help activate the transcription of sox 2 in NSCs.

\section{Brcal and Brm are recruited to the $R 1$ when OPCs convert to $2 A s$ and NSLCs}

To investigate whether Brcal and Brm are recruited to the R1 when OPCs are induced to convert to 2As and NSLCs, we fixed OPCs, 2As, and NSLCs in formaldehyde and used them for ChIP analysis as described above. As shown in Figure 5A, whereas neither anti-Brcal nor anti-Brm antibodies precipitated the R1 sequence in OPCs, both of them did so in 2As and NSLCs (Fig. 5A). This suggests that both Brcal and Brm are recruited to the R1 during the conversion of OPCs to 2As and NSLCs.

\section{A dominant-negative Brm decreases Sox2 expression in NSLCS}

To examine the relationship between Brm and sox2 expression, we constructed expression vectors encoding either GFP and wild-type human Brm (wBrm) or GFP and a dominant-negative form of human Brm (mutBrm), which has an inactive ATPase domain (Muchardt and Yaniv 1993; Muchardt et al. 1998; Bourachot et al. 1999). We transfected NSLCs with the vectors and immunolabeled the cells after $5 \mathrm{~d}$ for GFP and Sox2. Whereas all of the NSLCs transfected with wBrm expressed Sox2

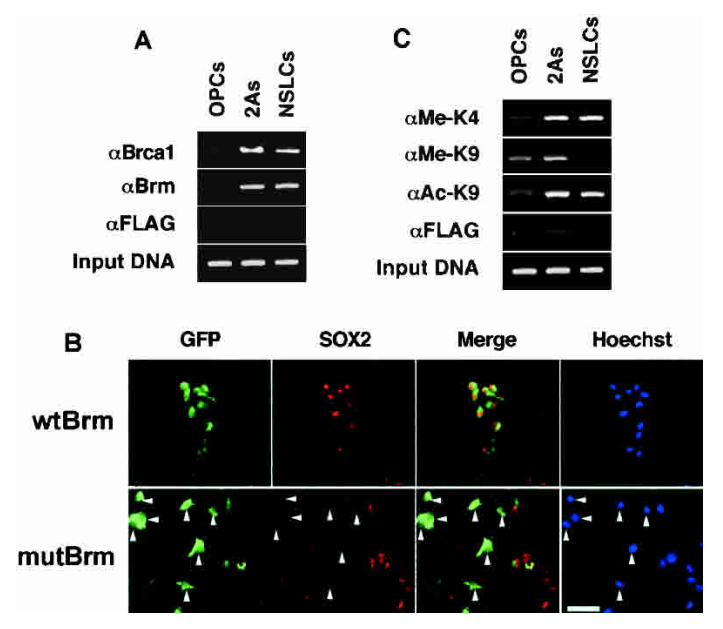

Figure 5. Recruitment of Brcal and Brm and histone $\mathrm{H} 3$ modification on the sox2 R1 enhancer during the conversion of OPCs to 2As and NSLCs. (A) ChIP analysis showing that R1 coprecipitates with Brcal or Brm. Extracts of formaldehyde-fixed OPCs, 2As, and NSLCs were precipitated with either anti-Brcal antibodies, anti-Brm antibodies, or anti-Flag antibody and Protein-A-Sepharose. Precipitated genomic DNA fragments were analyzed by PCR with the primer set for the R1 sequence. (B) NSLCs were transfected with the wBrm or mutBrm vector, cultured in bFGF for $3 \mathrm{~d}$, and then immunostained for both GFP (green) and Sox2 (in red). The Sox2-negative cells are indicated with arrowheads. Bar, $25 \mu \mathrm{m}$. (C) ChIP analysis showing the modifications of histone $\mathrm{H} 3$ associated with the R1 enhancer during OPC conversion. The cell extracts were precipitated with anti-dimethyl-H3-K4, anti-dimethyl-H3-K9, or antiacethyl-H3-K9 antibodies and Protein-A-Sepharose, and the precipitated genomic DNA fragments were analyzed as in $A$. 
(Fig. 5B), $<30 \%$ of the NSLCs transfected with mutBrm did so (Fig. 5B), suggesting that Brm and its ATPase-dependent chromatin-remodeling activity promotes the expression of sox2 in NSLCs.

\section{Histone H3 is modified when OPCs convert to $2 A s$} and NSLCS

There is increasing evidence that histone modification plays an important part in the control of gene expression during development (Heard et al. 2001; Litt et al. 2001; Lunyak et al. 2002; Song and Ghosh 2004). The acetylation (Ac) of histone $\mathrm{H} 3$ at $\mathrm{K} 9$ and its methylation (Me) at $\mathrm{K} 4$, for example, is associated with transcriptional activation, whereas the methylation of $\mathrm{H} 3$ at $\mathrm{K} 9$ is associated with transcriptional silencing. We examined histone $\mathrm{H} 3$ modification in the R1 enhancer of sox2 during the conversion of OPCs to NSLCs, using ChIP analysis. As shown in Figure 5C, antibodies specific for methylated $\mathrm{K} 9$ in $\mathrm{H} 3$ (Me-K9), but not antibodies specific for Me-K4 or Ac-K9, precipitated the R1 sequence from OPC extracts, consistent with the lack of expression of sox2 in these cells; in contrast, antibodies specific for either Me-K4 or Ac-K9 in $\mathrm{H} 3$ precipitated the R1 sequence from extracts of both $2 \mathrm{As}$ and NSLCs, consistent with the expression of sox 2 in both these cell types. Interestingly, antibodies specific for Me-K9 in $\mathrm{H} 3$ also precipitated the R1 sequence from extracts of 2 As but not from extracts of NSLCs, consistent with the increased expression of sox2 mRNA in NSLCs compared to 2As (see Fig. 1B). These findings suggest that histone $\mathrm{H} 3$ is progressively modified as OPCs convert to $2 \mathrm{As}$ and then NSLCs.

\section{Discussion}

In this study, we have examined some of the molecular changes that occur in OPCs when the cells are induced by extracellular signals to convert to $2 \mathrm{As}$ and then to NSLCs. One of the changes we find that is likely to be important is the induction of sox2. Although sox2 is not expressed in OPCs, it is expressed in 2As and NSLCs, as well as in NSCs, which are thought to give rise to OPCs. We have focused our attention on the chromosomal mechanisms involved in the re-expression of sox2 when OPCs convert to $2 \mathrm{As}$ and NSLCs. We provide evidence that the re-expression depends on the recruitment of chromatin-remodeling complexes to the R1 enhancer in the sox 2 promoter and is associated with modifications of $\mathrm{H} 3$ histones at this enhancer.

We show that OPCs rapidly acquire several molecular characteristics of NSCs when induced by BMPs to become 2As, which provides a plausible explanation for why this induction is apparently a required first step in the conversion of purified OPCs to NSLCs stimulated by extracellular signals (Kondo and Raff 2000a). Recently, it has been reported that newborn mouse (Belachew et al. 2003) and adult human (Nunes et al. 2003) OPCs can behave as NSCs in culture, even without purposeful 2A induction. In these studies, however, the OPCs were ex- posed to FCS, which contains BMPs (Kondo and Raff 2004); since even a brief exposure to BMPs is enough to induce 2A development (Mabie et al. 1997), it is possible that BMP-induced $2 \mathrm{~A}$ development occurred in these experiments.

We show here that Sox2, which is induced when OPCs convert to $2 \mathrm{As}$ and NSLCs, plays an important part in maintaining both the proliferation and undifferentiated state of NSLCs. When Sox2 is knocked down using RNAi, NSLCs stop proliferating, lose the expression of Nestin, and spontaneously differentiate into neurons. This finding is consistent with previous observations in vivo that overexpression of sox2 in mouse NSCs blocks their differentiation and that inhibition of Sox 2 in these cells causes their premature exit from the cell cycle and differentiation into neurons (Graham et al. 2003).

Sox 2 expression in both NSCs and NSLCs apparently depends on the tumor suppressor gene brca1, which is induced along with sox2 when OPCs convert to $2 \mathrm{As}$ and NSLCs. When Brcal levels are knocked down in these cells using RNAi, Sox2 protein is substantially decreased. In contrast, when Sox2 levels are knocked down in these cells, Brcal protein is not detectably decreased, suggesting that Brcal expression does not depend on Sox2. Although Sox2 expression in NSLCs and NSCs seems to depend on Brcal, overexpression of Brcal is not enough on its own to induce Sox 2 expression in OPCs, suggesting that other factors are also required.

Another protein that seems to be involved in Sox2 expression in NSLCs is Brm, an ATPase subunit in certain SWI/SNF chromatin-remodeling complexes. When a dominant-negative form of Brm, containing an inactive ATPase domain, is expressed in NSLCs, Sox 2 protein is substantially decreased, suggesting that the ATPase activity of Brm helps stimulate sox2 expression in these cells. Most importantly, we show using ChIP analysis that both Brcal and Brm are associated with the R1 enhancer sequence in the sox2 promoter in NSCs, 2As, and NSLCs, but not in OPCs; we show using a luciferase assay that this enhancer sequence is required for sox2-promoter-driven gene expression in NSCs. In coprecipitation assays, we demonstrate that at least some Brcal and Brm are in the same protein complexes in NSCs.

Taken together, these findings strongly suggest that SWI/SNF remodeling complexes containing Brm and Brcal are recruited to the $\mathrm{R} 1$ enhancer in the sox 2 promoter during the conversion of OPCs to 2As and NSLCs, where they help activate sox2 expression. It was shown previously that the expression of sox2 in the developing CNS depends on a patchwork of separate enhancers, each controlling a distinct spatial and temporal pattern of expression (Uchikawa et al. 2003). The R1 site that we identify here is contained in the enhancer that was previously found to be essential for sox2 expression in telencephalic NSCs in both mouse and chicken (Zappone et al. 2000; Uchikawa et al. 2003). Since both Brcal and Brm are expressed in proliferating NSCs in vivo and in vitro (Marquis et al. 1995; Korhonen et al. 2003; this 
study), it is likely that they both help activate sox2 expression in NSCs in vivo.

Interestingly, Brm-containing SWI/SNF complexes are also recruited to the promoters of both hes 1 and hes 5 genes in C2C12 myoblasts (Kadam and Emerson 2003), raising the possibility that these complexes might be required for stem cell maintenance in some nonneural tissues. Moreover, another chromatin-remodeling factor ISWI has been shown to help reprogram a somatic nucleus when it is transplanted into an unfertilized Xenopus egg (Kikyo et al. 2000), suggesting that chromatin remodeling may be a general feature of reprogramming in which cells (or nuclei) revert to a state with increased developmental potential.

We have also used ChIP analysis to show that $\mathrm{H} 3$ histones associated with the R1 sequence in the sox 2 promoter undergo progressive modification when OPCs are induced to convert to $2 \mathrm{As}$ and then NSLCs. The changes are consistent with the progressive activation of sox2 expression from OPCs-to-2As-to-NSLCs: in OPCs these $\mathrm{H} 3$ histones are in an inactive form, with $\mathrm{K} 9$ methylated and unacetylated and $\mathrm{K} 4$ unmethylated; in 2As they are in a more active form, with $\mathrm{K} 4$ methylated and K9 both methylated and acetylated, and in NSLCs they are in an even more active form, with K4 methylated and K9 acetylated but unmethylated. These changes are summarized in the schematic model shown in Figure 6.

In summary, we have shown that the conversion of OPCs to 2As and NSLCs is associated with extensive changes in gene expression, including the reactivation of sox2 expression. We have provided evidence that the reexpression of sox2 depends on chromatin remodeling by Brcal- and Brm-containing SWI/SNF complexes, which are recruited to the $\mathrm{R} 1$ enhancer in the sox2 promoter, and is associated with progressive modifications in $\mathrm{H} 3$ histones at this enhancer. It will be a major challenge to identify all the other genes and gene regulatory proteins involved in these conversions.

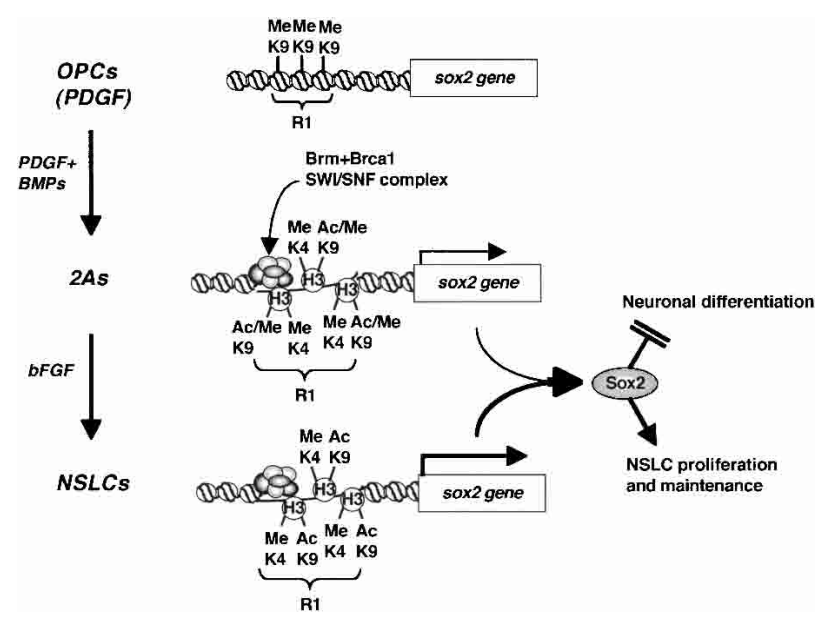

Figure 6. A model for how Brcal- and Brm-containing SWI/ SNF chromatin-remodeling complexes and histone $\mathrm{H} 3$ modifications may help activate sox2 transcription when OPCs are induced to convert to $2 \mathrm{As}$ and NSLCs.

\section{Materials and methods}

\section{Animals and chemicals}

Animals were obtained from the Animal Facilities at University College London and at University of Cambridge Centre for Brain Repair. Chemicals were purchased from Sigma, except where indicated. Recombinant human PDGF-AA, human BMP2, and human bFGF were purchased from Peprotech.

\section{Cell culture}

P6 rat optic nerve OPCs were purified to $>98 \%$ purity by sequential immunopanning as described previously (Barres et al. 1992) and cultured in poly-D-lysine (PDL)-coated 100-mm culture dishes (Falcon) in serum-free Dulbecco's Modified Eagle's medium containing bovine insulin $(10 \mu \mathrm{g} / \mathrm{mL})$, human transferrin $(100 \mu \mathrm{g} / \mathrm{mL})$, BSA $(100 \mu \mathrm{g} / \mathrm{mL})$, progesterone $(60 \mathrm{ng} / \mathrm{mL})$, putrescine $(16 \mu \mathrm{g} / \mathrm{mL})$, sodium selenite $(40 \mathrm{ng} / \mathrm{mL}), \mathrm{N}$-acetylcysteine $(60 \mu \mathrm{g} / \mathrm{mL})$, forskolin $(5 \mu \mathrm{M})$, PDGF $(10 \mathrm{ng} / \mathrm{mL})$, penicillin, and streptomycin (GIBCO; culture medium). To induce 2A differentiation, OPCs were cultured in PDL-coated culture dishes or slide flasks (Nunc) in the presence of PDGF and BMP2 (10 ng/mL) for 2-3 d as described before (Kondo and Raff 2004). To induce the conversion to NSLCs, 2As were cultured in the presence of bFGF (10 ng/mL) for 5-10 d in uncoated dishes. If cultures were maintained for longer than $4 \mathrm{~d}$, half of the medium was replaced every $2 \mathrm{~d}$.

NSCs were prepared from E14.5 mouse telencephalon as described before (Nakashima et al. 1999) and expanded in bFGF as floating spheres. For immunostaining, the spheres were cultured on poly-L-ornithine (PLO, $15 \mu \mathrm{g} / \mathrm{mL}$; SIGMA)- and fibronectin $(1 \mu \mathrm{g} / \mathrm{mL}$; Invitrogen)-coated eight-well chamber slides (Nunc) in the presence of bFGF for up to $5 \mathrm{~d}$, as described before (Nakashima et al. 1999).

\section{RT-PCR analysis}

RT-PCR was carried out as described previously (Kondo and Raff 2000b). Dimethylsulfoxide (DMSO) was added to the reaction mixture: 5\% DMSO for olig2, brca1, musashi1, and nestin, and 10\% DMSO for sox2 and id1-4. Cycle parameters for sox2, olig2, musashi1, hes1, hes5, bmi1, and nestin were $10 \mathrm{sec}$ at $94^{\circ} \mathrm{C}, 20 \mathrm{sec}$ at $58^{\circ} \mathrm{C}$, and $90 \mathrm{sec}$ at $72^{\circ} \mathrm{C}$ for 35 cycles. For gfap, brca1, id1-4, and bcrp1, they were $10 \mathrm{sec}$ at $94^{\circ} \mathrm{C}, 20 \mathrm{sec}$ at $58^{\circ} \mathrm{C}$, and $45 \mathrm{sec}$ at $72^{\circ} \mathrm{C}$ for 35 cycles. For $g 3 p d h$, they were 15 sec at $94^{\circ} \mathrm{C}, 30 \mathrm{sec}$ at $53^{\circ} \mathrm{C}$, and $90 \mathrm{sec}$ at $72^{\circ} \mathrm{C}$ for 23 cycles.

The following oligonucleotide DNA primers were synthesized: For sox2, the $5^{\prime}$ primer was 5'-ATGTATAACATGATG GAGACGGAGC- $3^{\prime}$, and the $3^{\prime}$ primer was $5^{\prime}$-TCACATGTGC GACAGGGGCAGTGT-3'. For $g f a p$, the $5^{\prime}$ primer was $5^{\prime}$-GA GATGATGGAGCTCAATGACC-3', and the $3^{\prime}$ primer was $5^{\prime}$ CTGGATCTCCTCCTCCAGCGA-3'. For olig2, the $5^{\prime}$ primer was $5^{\prime}$-ATGGACTCGGACGCCAGCCT-3', and the $3^{\prime}$ primer was $5^{\prime}$-TCACTTGGCGTCGGAGGTGAG-3'. For musashi1, the $5^{\prime}$ primer was $5^{\prime}$-CCTGGTTACACCTACCAGTTC-3', and the $3^{\prime}$ primer was 5'-TCAGTGGTACCCATTGGTGAAG-3'. For brca1, the $5^{\prime}$ primer was 5'-ATGGATTTATCTGCTGTTC GAATTC-3', and the $3^{\prime}$ primer was $5^{\prime}$-AAACCATTTGCA CACTGCATTCC-3'. For bmi1, the $5^{\prime}$ primer was $5^{\prime}$-ATG CATCGAACAACCAGAAT- $3^{\prime}$, and the $3^{\prime}$ primer was $5^{\prime}$ TCACTTTCCAGCTCTCCA-3'. For nestin, the $5^{\prime}$ primer was 5'-GCTACATACAGGACTCTGCTG-3', and the $3^{\prime}$ primer was 5'-AAACTCTAGACTCACTGGATTCT-3'. The primers for hes1, hes5, id1-4, bcrp1, and g3pdh were described previously (Kondo and Raff 2000b,c; Kondo et al. 2004). 


\section{Vector construction}

To deplete sox2 or brca1 mRNA and to mark the transfected cells, we constructed vectors based on the pMY vector (gift from T. Kitamura, University of Tokyo, Tokyo, Japan) (Misawa et al. 2000), which encodes green fluorescence protein ( $g f p$ ) and U6-promoter-driven siRNA for either sox2 (sox2-siRNA) or brca1 (brca1-siRNA). The synthesized sox2-siRNA oligonucleotides were 5'-TCGACAACCAAGACGCTCATGAAGT CAAGAGCTTCATGAGCGTCTTGGTTTTTTA- $3^{\prime}$ and 5' $^{\prime}$-AG CTTAAAAAAACCAAGACGCTCATGAAGCTCTTGACTT CATGAGCGTCTTGGTTG-3'. The synthesized brca1 siRNA oligonucleotides were 5'-TCGACAGGGCCTTCACAATGTC CTTTCAAGAGAAGGACATTGTGAAGGCCCTTTTTTT-3' and 5'-CTAGAAAAAAAGGGCCTTCACAATGTCCTTCTC TTGAAAGGACATTGTGAAGGCCCTG-3' .

To construct the series of sox2-enhancer-firefly luciferase expression vectors, mouse sox2 $5^{\prime}$ genomic DNA (gift from R. Lovell-Badge, NIMR, London, UK) was amplified and cloned into a pGL3-promoter vector (Promega), producing sox2-fullpGL3-pro (full), sox2-R1-pGL3-pro (R1), sox2-R2-pGL3-pro (R2), or sox2-R3-pGL3-pro (R3). The following oligonucleotide DNA primers were synthesized: For $R 1(-3833 \sim-3444)$, the $5^{\prime}$ primer was $5^{\prime}$-GTCAAATAGGGCCCTTTTCAG-3', and the 3' primer was 5'-AAGCCAACTGACAATGTTGTGG-3'. For $R 2(-3465 \sim-2684)$, the $5^{\prime}$ primer was $5^{\prime}$-CCACAACATTGTC AGTTGGCTT-3', and the $3^{\prime}$ primer was $5^{\prime}$-TAGTCTGAA CCTCTCCAATG-3'. For $R 3(-2704 \sim-1883)$, the $5^{\prime}$ primer was $5^{\prime}$-CATTGGAGAGGTTCAGACTA- ${ }^{\prime}$, and the $3^{\prime}$ primer was 5'-CTGCCCCAGGTTCTCCTTAAG-3'. Full sox2 5' genomic DNA (-3833 -1883) was amplified with R1 $5^{\prime}$ primer and R3 $3^{\prime}$ primer. The transcription start site is considered to be position +1 (Wiebe et al. 2000).

To determine whether Brm has a role in sox2 expression, we constructed vectors based on the pMY vector, which encodes GFP (to mark transfected cells) and either wild-type human Brm (wBrm) or mutant human Brm (mutBrm), which has an inactive ATPase domain (gift from C. Muchardt, Institut Pasteur, Paris, France).

\section{Transfection}

Transfection into OPCs and NSLCs was performed using the Nucleofector procedure according to the supplier's instructions (AMAXA). In brief, $2 \times 10^{6}$ cells were suspended in the Rat NSC Nucleofector Solution $(100 \mu \mathrm{L})$ with the expression vector $(10$ $\mu \mathrm{g})$, and the cells were transfected with the vector using the Nucleofector device. OPCs and NSLCs were then cultured in PDGF or bFGF, respectively.

To examine the effect of the transgenes, the transfected cells were harvested, and $5 \times 10^{3}$ cells per well were recultured in a PLO- and fibronectin-coated eight-well chamber slide (Nunc). To examine the effect on cell differentiation, the cells were cultured for $5 \mathrm{~d}$ in bFGF, fixed, and stained for neural markers (see below). To examine the effect on cell proliferation, $\mathrm{BrdU}(20$ $\mu \mathrm{M}$ ) was added after $5 \mathrm{~d} ; 8 \mathrm{~h}$ later, the cells were fixed and stained for BrdU (see below), and the proportion of $\mathrm{GFP}^{+}$cells that were $\mathrm{BrdU}^{+}$was determined.

\section{Immunocytochemistry}

Immunostaining was carried out as described previously (Kondo and Raff 2000a). The following antibodies were used to detect intracellular antigens: monoclonal anti-Nestin (Pharmingen; diluted 1:200), rabbit anti-Sox2 (gift from R. Lovell-Badge; diluted 1:500), rabbit anti-Brcal (Santa Cruz; diluted 1:200), mono- clonal anti-GFAP (Sigma; 1:200), rabbit anti-GFAP (DACO; diluted 1:400), a mixture of rabbit anti-NF antibodies (Affiniti; diluted 1:100), monoclonal anti-GFP, and rabbit anti-GFP (Molecular Probe; diluted 1:200). To detect surface antigens, we used the A2B5 monoclonal antibody to detect OPCs (hybridoma supernatant; diluted 1:5) (Eisenbarth et al. 1979), monoclonal anti-PSA-NCAM (gift from T. Seki, University of Tokyo, Tokyo, Japan; diluted 1:1000), and monoclonal anti-SSEA1 (Santa Cruz; diluted 1:200). The antibodies were detected with TexasRed-conjugated goat anti-mouse IgG or IgM (Jackson Immunoresearch; diluted 1:200), fluorescein-conjugated goat anti-rabbit IgG (Jackson Immunoresearch; diluted 1:200), and Alexa488-coupled goat anti-mouse IgG and Alexa-594-coupled goat anti-rabbit IgG (Molecular Probe; diluted 1:200). Cells were stained for BrdU as previously described (Kondo and Raff 2000b), by using a monoclonal anti-BrdU antibody (hybridoma culture supernatant; diluted 1:5) (Magaud et al. 1988), followed by Texas-Red-conjugated goat anti-mouse IgG. To visualize all nuclei, the cells were counterstained with bisbenzimide (Hoechst 33342; $1 \mu \mathrm{g} / \mathrm{mL})$.

\section{Luciferase assay}

NSCs were cultured on PLO- and fibronectin-coated six-well plates (Nunc). On the following day, cells were transfected with $2 \mu \mathrm{g}$ of the full, R1, R2, or R3 vectors encoding firefly luciferase and $0.2 \mu \mathrm{g}$ of the internal control vector pEF-Rluc (gift from S. Nagata and K. Shimozaki, Osaka University, Osaka, Japan) encoding sea pansy luciferase using the Superfect Transfection Reagent according to the supplier's instructions (QIAGEN). After $24 \mathrm{~h}$, the activities of the two types of luciferase were measured using the Dual-Luciferase Reporter Assay System according to the supplier's instructions (Promega).

\section{ChIP assay}

ChIP assays were performed as described in Shang et al. (2000). In brief, we fixed $2 \times 10^{6}$ NSCs, OPCs, 2 As, or NSLCs in $4 \%$ formaldehyde, suspended them in a cell lysis buffer (1\% SDS, 10 $\mathrm{mM}$ EDTA, $50 \mathrm{mM}$ Tris- $\mathrm{HCl}$ at $\mathrm{pH} 8.1,5 \mathrm{mg} / \mathrm{mL}$ aprotinin, and $3 \mathrm{mM}$ PMSF), sonicated them, and harvested cell extracts by centrifugation. The extracts were incubated with antibodies overnight and then immunoprecipitated with Protein-A-Sepharose (50 $\mu \mathrm{L}$ of $50 \%$ suspension). The following antibodies were used for the assay; anti-Brcal antibodies $(10 \mu \mathrm{g})$, anti-Brm antibodies (Santa Cruz; $2 \mu \mathrm{g}$ ), anti-Brg1 antibodies (Santa Cruz; 2 $\mu \mathrm{g})$, anti-dimethyl-histone H3-K4 antibodies (UPSTATE; 1:100), anti-dimethyl-histone H3-K9 antibodies (UPSTATE; 1:100), and anti-acethyl-histone H3-K9 antibodies (UPSTATE; 1:100). Precipitates were heated to reverse the formaldehyde cross-linking. The DNA fragments in the precipitates were purified by phenol/ chloroform extraction and $\mathrm{EtOH}$ precipitation and then used for PCR with primer sets for R1, R2, and R3. Cycle parameters were $10 \mathrm{sec}$ at $94^{\circ} \mathrm{C}, 20 \mathrm{sec}$ at $60^{\circ} \mathrm{C}$, and $90 \mathrm{sec}$ at $72^{\circ} \mathrm{C}$ for 30 cycles.

\section{Immunoprecipitation}

Immunoprecipitation assays were performed as described in Fukuda et al. (2004). In brief, $2 \times 10^{6}$ NSCs were harvested, suspended in NP-40 lysis buffer (10 mM Tris- $\mathrm{HCl}$ at $\mathrm{pH} 7.5,150$ $\mathrm{mM} \mathrm{NaCl}, 0.5 \% \mathrm{NP}-40,5 \mathrm{mM}$ EDTA, $5 \mathrm{mg} / \mathrm{mL}$ aprotinin, and $3 \mathrm{mM}$ PMSF), and sonicated. After centrifugation, cell extracts were immunoprecipitated with anti-Flag M2, anti-Brca1, or anti-Brm antibodies and Protein-A-Sepharose. Immunoprecipitates were analyzed using anti-Brm antibodies. 


\section{Acknowledgments}

We thank Robin Lovell-Badge for mouse sox2 5' genomic DNA and rabbit anti-SOX2 antibodies, Koji Shimozaki and Shigekazu Nagata for the pEF-Rluc vector, Toshio Kitamura for the pMY vector, Christian Muchardt for CMV-HA-wBrm and CMV-HAmutBrm, and Tatsunori Seki for the mouse monoclonal antiPSA-NCAM antibody. T.K. was partly supported by Merck Sharp and Dohme, and M.R. was supported by the Medical Research Council, UK.

\section{References}

Akazawa, C., Sasai, Y., Nakanishi, S., and Kageyama, R. 1992. Molecular characterization of a rat negative regulator with a basic helix-loop-helix structure predominantly expressed in the developing nervous system. J. Biol. Chem. 267: 2187921885.

Avilion, A.A., Nicolis, S.K., Pevny, L.H., Perez, L., Vivian, N., and Lovell-Badge, R. 2003. Multipotent cell lineages in early mouse development depend on SOX2 function. Genes \& Dev. 17: 126-140.

Barres, B.A. and Raff, M.C. 1994. Control of oligodendrocyte number in the developing rat optic nerve. Neuron 12: 935942.

Barres, B., Hart, I., Coles, H., Burne, J., Voyvodic, J., Richardson, W., and Raff, M. 1992. Cell death and control of cell survival in the oligodendrocyte lineage. Cell 70: 31-46.

Barres, B., Lazar, M., and Raff, M. 1994. A novel role for thyroid hormone, glucocorticoids and retinoic acid in timing oligodendrocyte development. Development 120: 1097-1108.

Belachew, S., Chittajallu, R., Aguirre, A.A., Yuan, X., Kirby, M., Anderson, S., and Gallo, V. 2003. Postnatal NG2 proteoglycan-expressing progenitor cells are intrinsically multipotent and generate functional neurons. J. Cell Biol. 161: 169-186.

Benezra, R., Davis, R.L., Lockshon, D., Turner, D.L., and Weintraub, H. 1990. The protein Id: A negative regulator of helixloop-helix DNA binding proteins. Cell 61: 49-59.

Biggs, J., Murphy, E.V., and Israel, M.A. 1992. A human Id-like helix-loop-helix protein expressed during early development. Proc. Nat1. Acad. Sci. 89: 1512-1516.

Bignami, A., Eng, L.F., Dahl, D., and Uyeda, C.T. 1972. Localization of the glial fibrillary acidic protein in astrocytes by immunofluorescence. Brain Res. 43: 429-435.

Bochar, D.A., Wang, L., Beniya, H., Kinev, A., Xue, Y., Lane, W.S., Wang, W., Kashanchi, F., and Shiekhattar, R. 2000. BRCA1 is associated with a human SWI/SNF-related complex: Linking chromatin remodeling to breast cancer. Cell 102: 257-265.

Bourachot, B., Yaniv, M., and Muchardt, C. 1999. The activity of mammalian brm/SNF2a is dependent on a high-mobilitygroup protein I/Y-like DNA binding domain. Mol. Cell. Biol. 19: 3931-3939.

Bylund, M., Andersson, E., Novitch, B.G., and Muhr, J. 2003. Vertebrate neurogenesis is counteracted by Sox $1-3$ activity. Nat. Neurosci. 6: 1162-1168.

Capela, A. and Temple, S. 2002. LeX/ssea-1 is expressed by adult mouse CNS stem cells, identifying them as nonependymal. Neuron 35: 865-875.

Christy, B.A., Sanders, L.K., Lau, L.F., Copeland, N.G., Jenkins, N.A., and Nathans, D. 1991. An Id-related helix-loop-helix protein encoded by a growth factor-inducible gene. Proc. Natl. Acad. Sci. 88: 1815-1819.

Dawson, M.R., Polito, A., Levine, J.M., and Reynolds, R. 2003. NG2-expressing glial progenitor cells: An abundant and widespread population of cycling cells in the adult rat CNS.
Mol. Cell. Neurosci. 24: 476-488.

Doetsch, F., Petreanu, L., Caille, I., Garcia-Verdugo, J.M., and Alvarez-Buylla, A. 2002. EGF converts transit-amplifying neurogenic precursors in the adult brain into multipotent stem cells. Neuron 36: 1021-1034.

Doyle, L.A., Yang, W., Abruzzo, L.V., Krogmann, T., Gao, Y., Rishi, A.K., and Ross, D.D. 1999. A multidrug resistance transporter from human MCF-7 breast cancer cells. Proc. Natl. Acad. Sci. 95: 15665-15670.

Eisenbarth, G.S., Walsh, F.S., and Nirenburg, M. 1979. Monoclonal antibodies to a plasma membrane antigen of neurons. Proc. Natl. Acad. Sci. 76: 4913-4916.

Fukuda, S., Kondo, T., Takebayashi, H., and Taga, T. 2004. Negative regulatory effect of an oligodendrocytic bHLH factor OLIG2 on the astrocytic differentiation pathway. Cell Death Differ. 11: 196-202.

Graham, V., Khudyakov, J., Ellis, P., and Pevny, L. 2003. SOX2 functions to maintain neural progenitor identity. Neuron 39: 749-765.

Gubbay, J., Collignon, J., Koopman, P., Capel, B., Economou, A., Munsterberg, A., Vivian, N., Goodfellow, P., and LovellBadge, R. 1990. A gene mapping to the sex-determining region of the mouse $\mathrm{Y}$ chromosome is a member of a novel family of embryonically expressed genes. Nature 346: 245250.

Hakem, R., de la Pompa, J.L., Sirard, C., Mo, R., Woo, M., Hakem, A., Wakeham, A., Potter, J., Reitmair, A., Billia, F., et al. 1996. The tumor suppressor gene Brcal is required for embryonic cellular proliferation in the mouse. Cell 85: 1009-1023.

Heard, E., Rougeulle, C., Arnaud, D., Avner, P., Allis, C.D., and Spector, D.L. 2001. Methylation of histone H3 at Lys-9 is an early mark on the $\mathrm{X}$ chromosome during $\mathrm{X}$ inactivation. Cell 107: 727-738.

Julien, J.P. 1999. Neurofilament functions in health and disease. Curr. Opin. Neurobiol. 9: 554-560.

Kadam, S. and Emerson, B.M. 2003. Transcriptional specificity of human SWI/SNF BRG1 and BRM chromatin remodeling complexes. Mol. Cell 11: 377-389.

Kikyo, N., Wade, P.A., Guschin, D., Ge, H., and Wolffe, A.P. 2000. Active remodeling of somatic nuclei in egg cytoplasm by the nucleosomal ATPase ISWI. Science 289: 2360-2362.

Kondo, T. and Raff, M. 2000a. Oligodendrocyte precursor cells reprogrammed to become multipotential CNS stem cells. Science 289: 1754-1757.

. 2000b. The Id 4 HLH protein and the timing of oligodendrocyte differentiation. EMBO J. 19: 1998-2007.

- 2000c. Basic helix-loop-helix proteins and the timing of oligodendrocyte differentiation. Development 127: 29892998.

- 2004. A role for Noggin in the development of oligodendrocyte precursor cells. Dev. Biol. 267: 242-251.

Kondo, T., Setoguchi, T., and Taga, T. 2004. Persistence of a small subpopulation of cancer stem-like cells in the C6 glioma cell line. Proc. Natl. Acad. Sci. 101: 781-786.

Korhonen, L., Brannvall, K., Skoglosa, Y., and Lindholm, D. 2003. Tumor suppressor gene BRCA-1 is expressed by embryonic and adult neural stem cells and involved in cell proliferation. J. Neurosci. Res. 71: 769-776.

Lendahl, U., Zimmerman, L.B., and McKay, R.D. 1990. CNS stem cells express a new class of intermediate filament protein. Cell 60: 585-595.

Li, M., Pevny, L., Lovell-Badge, R., and Smith, A. 1998. Generation of purified neural precursors from embryonic stem cells by lineage selection. Curr. Biol. 8: 971-974.

Litt, M.D., Simpson, M., Gaszner, M., Allis, C.D., and Felsen- 
feld, G. 2001. Correlation between histone lysine methylation and developmental changes at the chicken $\beta$-globin locus. Science 293: 2453-2455.

Liu, C.Y., Flesken-Nikitin, A., Li, S., Zeng, Y., and Lee, W.H. 1996. Inactivation of the mouse Brcal gene leads to failure in the morphogenesis of the egg cylinder in early postimplantation development. Genes \& Dev. 10: 1835-1843.

Lu, Q.R., Yuk, D., Alberta, J.A., Zhu, Z., Pawlitzky, I., Chan, J., McMahon, A.P., Stiles, C.D., and Rowitch, D.H. 2000. Sonic hedgehog-regulated oligodendrocyte lineage genes encoding bHLH proteins in the mammalian central nervous system. Neuron 25: 317-329.

Lunyak, V.V., Burgess, R., Prefontaine, G.G., Nelson, C., Sze, S.H., Chenoweth, J., Schwartz, P., Pevzner, P.A., Glass, C., Mandel, G., et al. 2002. Corepressor-dependent silencing of chromosomal regions encoding neuronal genes. Science 298: $1747-1752$.

Mabie, P.C., Mehler, M.F., Marmur, R., Papavasiliou, A., Song, Q., and Kessler, J.A. 1997. Bone morphogenetic proteins induce astroglial differentiation of oligodendroglial-astroglial progenitor cells. I. Neurosci. 17: 4112-4120.

Magaud, J.P., Sargent, I., and Mason, D.Y. 1988. Detection of human white cell proliferative responses by immunoenzymatic measurement of bromodeoxyuridine uptake. J. Immunol. Methods 106: 95-100.

Marquis, S.T., Rajan, J.V., Wynshaw-Boris, A., Xu, J., Yin, G.Y., Abel, K.J., Weber, B.L., and Chodosh, L.A. 1995. The developmental pattern of Brcal expression implies a role in differentiation of the breast and other tissues. Nat. Genet. 11: 17-26.

Miki, Y., Swensen, J., Shattuck-Eidens, D., Futreal, P.A., Harshman, K., Tavtigian, S., Liu, Q., Cochran, C., Bennett, L.M., Ding, W., et al. 1994. A strong candidate for the breast and ovarian cancer susceptibility gene BRCA1. Science 266: 6671.

Misawa, K., Nosaka, T., Morita, S., Kaneko, A., Nakahata, T., Asano, S., and Kitamura, T. 2000. A method to identify cDNAs based on localization of green fluorescent protein fusion products. Proc. Natl. Acad. Sci. 97: 3062-3066.

Muchardt, C. and Yaniv, M. 1993. A human homologue of Saccharomyces cerevisiae SNF2/SWI2 and Drosophila brm genes potentiates transcriptional activation by the glucocorticoid receptor. EMBO J. 12: 4279-4290.

Muchardt, C., Bourachot, B., Reyes, J.-C., and Yaniv, M. 1998. ras transformation is associated with decreased expression of the brm/SNF2 $\alpha$ ATPase from the mammalian SWI-SNF complex. EMBO J. 17: 223-231.

Nakamura, M., Okano, H., Blendy, J.A., and Montell, C. 1994. Musashi, a neural RNA-binding protein required for Drosophila adult external sensory organ development. Neuron 13: $67-81$.

Nakashima, K., Yanagisawa, M., Arakawa, H., Kimura, N., Hisatsune, T., Kawabata, M., Miyazono, K., and Taga, T. 1999. Synergistic signaling in fetal brain by STAT3-Smad1 complex bridged by p300. Science 284: 479-482.

Nunes, M.C., Roy, N.S., Keyoung, H.M., Goodman, R.R., McKhann II, G., Jiang, L., Kang, J., Nedergaard, M., and Goldman, S.A. 2003. Identification and isolation of multipotential neural progenitor cells from the subcortical white matter of the adult human brain. Nat. Med. 9: 439-447.

Raff, M.C., Mirsky, R., Fields, K.L., Lisak, R.P., Dorfman, S.H., Silberberg, D.H., Gregson, N.A., Leibowitz, S., and Kennedy, M.C. 1978. Galactocerebroside is a specific cell-surface antigenic marker for oligodendrocytes in culture. Nature 274: 813-816.
Raff, M.C., Miller, R.H., and Noble, M. 1983. A glial progenitor cell that develops in vitro into an astrocyte or an oligodendrocyte depending on culture medium. Nature 303: 390-396.

Riechmann, V., van Cruchten, I., and Sablitzky, F. 1994. The expression pattern of Id4, a novel dominant negative helixloop-helix protein, is distinct from Id1, Id2 and Id3. Nucleic Acids Res. 22: 749-755.

Sasai, Y., Kageyama, R., Tagawa, Y., Shigemoto, R., and Nakanishi, S. 1992. Two mammalian helix-loop-helix factors structurally related to Drosophila hairy and Enhancer of split. Genes \& Dev. 6: 2620-2634.

Seki, T. and Arai, Y. 1991. The persistent expression of a highly polysialylated NCAM in the dentate gyrus of the adult rat. Neurosci. Res. 12: 503-513.

Shang, Y., Hu, X., DiRenzo, J., Lazar, M.A., and Brown, M. 2000. Cofactor dynamics and sufficiency in estrogen receptorregulated transcription. Cell 103: 843-852.

Song, M.R. and Ghosh, A. 2004. FGF2-induced chromatin remodeling regulates CNTF-mediated gene expression and astrocyte differentiation. Nat. Neurosci. 7: 229-235.

Sun, X.H., Copeland, N.G., Jenkins, N.A., and Baltimore, D. 1991. Id proteins Id 1 and Id2 selectively inhibit DNA binding by one class of helix-loop-helix proteins. Mol. Cell. Biol. 11: 5603-5611.

Takebayashi, H., Yoshida, S., Sugimori, M., Kosako, H., Kominami, R., Nakafuku, M., and Nabeshima, Y. 2000. Dynamic expression of basic helix-loop-helix Olig family members: Implication of Olig2 in neuron and oligodendrocyte differentiation and identification of a new member, Olig3. Mech. Dev. 99: 143-148.

Uchikawa, M., Ishida, Y., Takemoto, T., Kamachi, Y., and Kondoh, H. 2003. Functional analysis of chicken Sox2 enhancers highlights an array of diverse regulatory elements that are conserved in mammals. Dev. Cell 4: 509-519.

van Lohuizen, M., Verbeek, S., Scheijen, B., Wientjens, E., van der Gulden, H., and Berns, A. 1991. Identification of cooperating oncogenes in E mu-myc transgenic mice by provirus tagging. Cell 65: 737-752.

Wiebe, M.S., Wilder, P.J., Kelly, D., and Rizzino, A. 2000. Isolation, characterization, and differential expression of the murine Sox-2 promoter. Gene 246: 383-393.

Zappone, M.V., Galli, R., Catena, R., Meani, N., De Biasi, S., Mattei, E., Tiveron, C., Vescovi, A.L., Lovell-Badge, R., Ottolenghi, S., et al. 2000. Sox2 regulatory sequences direct expression of a $\beta$-geo transgene to telencephalic neural stem cells and precursors of the mouse embryo, revealing regionalization of gene expression in CNS stem cells. Development 127: 2367-2382.

Zhou, Q., Wang, S., and Anderson, D.J. 2000. Identification of a novel family of oligodendrocyte lineage-specific basic helixloop-helix transcription factors. Neuron 25: 331-343. 


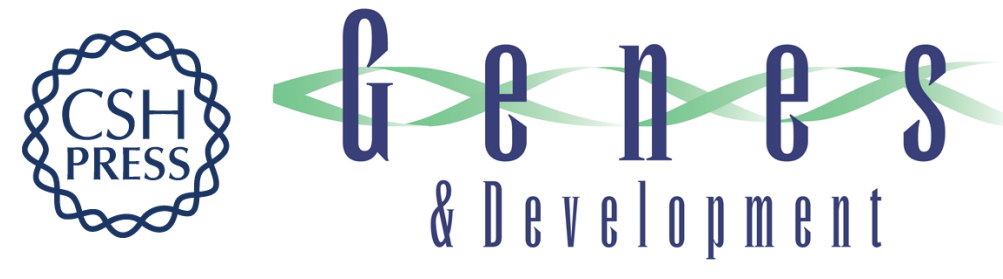

\section{Chromatin remodeling and histone modification in the conversion of oligodendrocyte precursors to neural stem cells}

Toru Kondo and Martin Raff

Genes Dev. 2004, 18:

Access the most recent version at doi:10.1101/gad.309404

References This article cites 61 articles, 24 of which can be accessed free at: http://genesdev.cshlp.org/content/18/23/2963.full.html\#ref-list-1

License

Email Alerting

Receive free email alerts when new articles cite this article - sign up in the box at the top Service right corner of the article or click here.

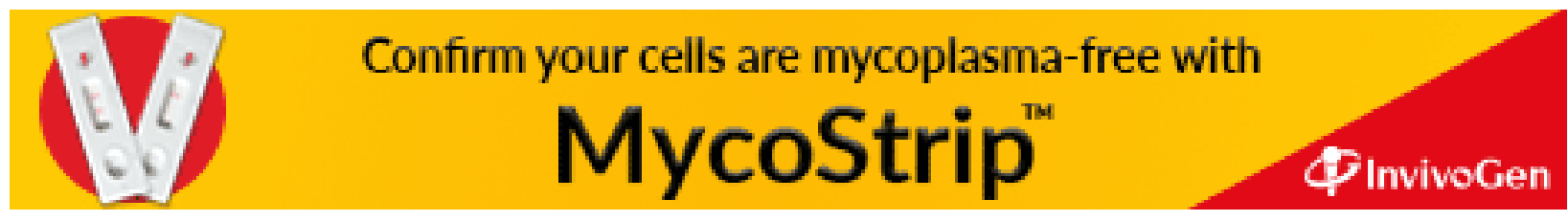

